\title{
Weekend effect in extracorporeal membrane oxygenation therapy initiation: a nationwide cohort study in South Korea
}

\author{
Hyoung-Won Cho ${ }^{1 \#}$, In-Ae Song ${ }^{2 \#}$, Tak Kyu $\mathrm{Oh}^{2} \wedge$ \\ ${ }^{1}$ Department of Cardiology, Cardiovascular Center, Seoul National University Bundang Hospital, Seongnam, South Korea; ${ }^{2}$ Department of \\ Anesthesiology and Pain Medicine, Seoul National University Bundang Hospital, Seongnam, South Korea \\ Contributions: (I) Conception and design: TK Oh, IA Song; (II) Administrative support: IA Song, HW Cho; (III) Provision of study materials or \\ patients: All authors; (IV) Collection and assembly of data: TK Oh, IA Song; (V) Data analysis and interpretation: TK Oh; (VI) Manuscript writing: \\ All authors; (VII) Final approval of manuscript: All authors. \\ \#These authors contributed equally to this work. \\ Correspondence to: Tak Kyu Oh. Department of Anesthesiology and Pain Medicine, Seoul National University Bundang Hospital, Gumi-ro 173 Beon- \\ gil, Bundang-gu, Seongnam 13620, South Korea. Email: airohtak@hotmail.com.
}

Background: Extracorporeal membrane oxygenation (ECMO) therapy requires close monitoring and optimal management after initiation. However, it remains unclear whether the day of the week of ECMO initiation affects patient outcomes. We aimed to investigate whether the initiation of ECMO therapy during a weekend was associated with increased mortality risk.

Methods: We performed a population-level cohort study, based on health records obtained from the National Health Insurance Service database in South Korea. All critically ill adult patients who received ECMO therapy in the intensive care unit during 2005-2018 were enrolled. The primary endpoint was the 60-day mortality rate following ECMO therapy. Multivariable Cox regression was used for the analysis.

Results: Data from a total of 21,129 ECMO patients were included in the analysis, of whom 12,825 (60.7\%) died within 60 days. There were 4,647 (22.0\%) patients who received ECMO therapy during a weekend (weekend group). Multivariable Cox regression revealed that the weekend group was at increased risk of 60-day mortality compared to the weekday group (hazard ratio: 1.05, 95\% confidence interval: 1.01 to 1.09 ; $\mathrm{P}=0.025$ ). In the sensitivity analysis, ECMO patients whose therapy started on a Saturday were at higher risk of 60-day mortality (hazard ratio relative to those whose therapy started on a Wednesday: 1.11, 95\% confidence interval: 1.04-1.19; $\mathrm{P}=0.003$ ). However, starting ECMO therapy on other weekdays (Thursday, Friday, Sunday, Monday, or Tuesday) did not affect mortality outcomes ( $>>0.05)$.

Conclusions: ECMO therapy initiated during a weekend was associated with a slightly increased risk of 60-day mortality compared to that associated with ECMO therapy initiated on a weekday. This association was more evident in patients who received ECMO therapy that started on a Saturday. Further studies are needed to confirm these findings.

Keywords: Extracorporeal membrane oxygenation (ECMO); critical care; intensive care units; mortality; cohort studies

Submitted Jan 13, 2021. Accepted for publication Mar 17, 2021.

doi: $10.21037 /$ atm-21-180

View this article at: http://dx.doi.org/10.21037/atm-21-180

^ ORCID: 0000-0002-4027-4423. 


\section{Introduction}

Extracorporeal membrane oxygenation (ECMO) is an artificial extracorporeal support system that is used to treat refractory cardiac and/or respiratory failure $(1,2)$. The clinical indications for ECMO use include heart failure, pulmonary hypertension, myocarditis, post-cardiac surgery management, intractable arrhythmia, trauma, shock, respiratory failure, and acute respiratory distress syndrome (ARDS) (3-7). Recently, ECMO has been used as rescue therapy in patients with coronavirus disease $(8,9)$, and the number of potential indications for its use in intensive care units (ICUs) continues to expand (10).

The "weekend effect" is a phenomenon wherein increased mortality rates are observed among patients admitted to a hospital on Saturdays and/or Sundays (off-hours) (11). This effect has been attributed to the reduced size of hospital staff during weekends (12), and greater illness severity in patients that require an off-hours admission (13). Previous studies of critically ill patients have shown an association between ICU admission during a weekend and increased risk of inhospital mortality $(14,15)$. In South Korea, only $17.3 \%$ and $29.4 \%$ of ICUs had intensive care specialists with a 5-day work week in 2009 and 2014, respectively, and most ICUs did not have intensivist staffing on weekends (16). Therefore, the "weekend effect" might exist in the ICUs of South Korea.

The initiation of ECMO therapy may cause immediate, complex, and systemic inflammatory reactions that are similar to those seen in systemic inflammatory response syndrome (17). These inflammatory reactions, if severe, can cause endothelial injury; disruption of microcirculation; and major organ injuries, such as severe kidney injury, pneumonia, and liver dysfunction (18). Moreover, ECMO patients usually require fluid resuscitation or blood transfusion due to hypotension or bleeding after the initiation of ECMO therapy (19). Thus, the fluid balance, electrolyte loss, and nutritional status of ECMO patients require close monitoring and proper management in the first 1-2 days after the initiation of ECMO therapy (19). Accordingly, starting ECMO therapy on a weekend may affect patient outcomes. Although the weekend effect has been reported in patients that underwent extracorporeal cardiopulmonary resuscitation (20), no previous study has examined whether patient outcomes associated with the initiation of ECMO therapy are also subject to this effect. In South Korea, the National Health Insurance Service
(NHIS) maintains electronic health records with regard to ECMO therapy.

Using the NHIS database, we aimed to investigate whether the initiation of ECMO therapy during a weekend was associated with increased mortality risk among ECMO patients. We hypothesized that starting ECMO therapy on weekends was associated with mortality risk higher than that associated with starting ECMO on weekdays. We present the following article in accordance with the STROBE reporting checklist (21) (available at http://dx.doi. org/10.21037/atm-21-180).

\section{Methods}

The study was a population-level cohort study. The study was conducted in accordance with the Declaration of Helsinki (as revised in 2013), and was approved by Institutional Review Board of Seoul National University Bundang Hospital (X-2001-586-902). The informed consent requirement was waived, as the data were anonymized and analyzed retrospectively, using a dataset extracted from the South Korean NHIS database. The data were extracted by an independent medical records technician (SY Park) at the NHIS center who had no conflicts of interest associated with this study. In South Korea, all the disease diagnosisand prescription-related information corresponding to medications and/or procedures, including surgeries, should be registered in the NHIS database by physicians in order for the patients to receive proper financial coverage of any treatment charges.

\section{ECMO patients}

All critically ill adult patients (age $\geq 18$ years old) who had received ECMO therapy in the ICU (outside the operating room), following hospitalization during 2005-2018 were included in this study. The codes related to ECMO use in the NHIS database were O1901-1904. Cases involving Novalung therapy (O1905) were not considered as ECMO therapy cases in this study.

\section{Exposure: weekend initiation of ECMO therapy}

ECMO patients whose therapy started on a weekend (Saturday or Sunday) were defined as the "weekend" group. ECMO patients whose therapy started on a weekday (Monday-Friday) were defined as the "weekday" group. 


\section{Primary endpoints: 60-day mortality}

The primary endpoint was the 60-day mortality rate, which was defined as any death within 60 days after the date of ECMO therapy initiation. The 60-day mortality rate was set as the primary endpoint based on a previous study (2) because some ECMO patients, including those who were waiting for heart or lung transplantation, received ECMO therapy for long periods. Data on the exact dates of death of all patients who had received ECMO therapy until December 31, 2019 were extracted.

\section{Covariates}

The following information was extracted as covariates of interest: demographic information (age and sex) and socioeconomic characteristics (area of residence and annual income level); and Charlson Comorbidity Index (CCI) scores, which were calculated using the International Classification of Diseases, 10th revision, codes assigned during a period of 1 year prior to the start date of ECMO therapy, as shown in Table S1. In addition, treatmentrelated information was extracted, including the duration of hospitalization and ECMO therapy (days), and the main indication for initiating ECMO therapy. The main diagnoses at the initiation of ECMO therapy were extracted and classified into nine groups: cardiovascular disease, ARDS or respiratory failure, post-cardiac arrest, cancer, gastrointestinal tract disease, sepsis, shock, trauma, burn and injury, and others.

The volume of cases involving ECMO therapy during the 14-year study period was used to determine the treatment capacity of the ECMO centers in South Korea; previous studies have suggested this capacity to be a factor affecting ECMO therapy outcomes (22). The volume of cases involving ECMO therapy was defined as the sum of the total number of cases involving ECMO therapy administered to adults during the 14-year period at each hospital. This was divided into four groups, using quartile ratios (Q1, <190; Q2, 190-393; Q3, 394-883; and Q4, $>883)$.

\section{Statistical analysis}

First, we assessed the normality of continuous variables (age, CCI, duration of hospitalization, and duration of ECMO therapy) using the Kolmogorov-Smirnov test. As a result, the continuous variables did not follow the normal distribution. Accordingly, the clinico-epidemiological characteristics of the patients who underwent ECMO therapy were presented as median values with interquartile range (IQR) for continuous variables, and counts with percentages for categorical variables. To compare the clinico-epidemiological characteristics between the weekend and control groups, the Mann-Whitney U test and chi-square test were used for continuous and categorical variables, respectively. Next, we performed univariable and multivariable Cox regression analyses to investigate whether the initiation of ECMO therapy on weekends was associated with an increased risk of 60-day mortality. In this time-toevent analysis, the survival time was set as the duration, and death within 60 days was considered the event of interest. All the covariates were included in the multivariable Cox regression model. The absence of multicollinearity in all the multivariable models was confirmed by a variance inflation factor of $<2.0$.

As a sensitivity analysis, another multivariable Cox regression model was fitted to investigate the association between the initiation of ECMO therapy on a specific day of the week and 60-day mortality risk. Specifically, the risk of 60-day mortality among patients who received ECMO therapy on any day other than Wednesday was compared with that of patients who received ECMO therapy on a Wednesday. The results of the Cox regression were presented as hazard ratios (HRs) with $95 \%$ confidence intervals (CIs), and C-statistics were used to identify the C-index of the multivariable Cox regression model. In addition, a log-minus-log plot was used to confirm that the Cox proportional hazards assumption was satisfied. All statistical analyses were performed using $\mathrm{R}$ software (version 4.0.3 with R packages, the R Project for Statistical Computing, Vienna, Austria). $\mathrm{P}$ values of $<0.05$ were considered indicative of a statistically significant finding.

\section{Results}

From January 1, 2005, to December 31, 2018, a total of 22,793 critically ill patients received ECMO therapy at 128 ECMO centers in South Korea. After excluding 1,664 patients aged $<18$ years, data from a total of 21,129 patients were included in the analysis, of whom $12,825(60.7 \%)$ died within 60 days of initiating therapy (Figure 1). The clinico-epidemiological characteristics of the cohort are presented in Table 1. The patients received ECMO therapy for $8.2 \pm 13.7$ days, and were hospitalized for $17.4 \pm 15.8$ days. The number of ECMO patients in the 


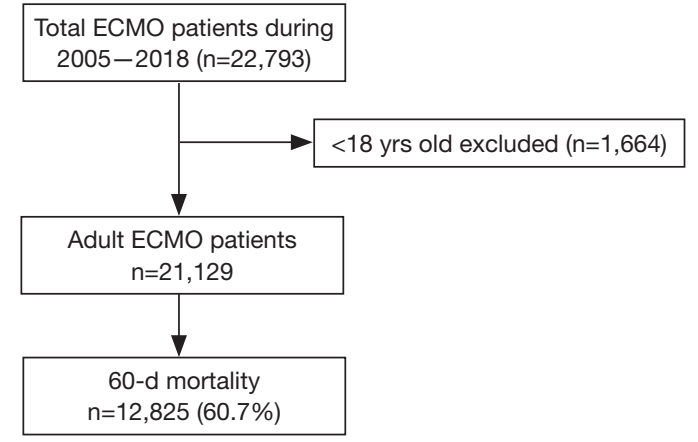

Figure 1 Flow chart depicting ECMO patient selection. ECMO, extracorporeal membrane oxygenation

Table 1 The clinico-epidemiological characteristics of the overall patients

\begin{tabular}{|c|c|}
\hline Variable & Number (\%) \\
\hline Age, year, median [IQR] & $61.0[50,70]$ \\
\hline Sex, male & $13,986(66.2)$ \\
\hline \multicolumn{2}{|l|}{ Residence at ECMO treatment } \\
\hline Capital city (Seoul) & 4,993 (23.6) \\
\hline Other metropolitan city & $4,844(22.9)$ \\
\hline Other area & $11,292(53.4)$ \\
\hline \multicolumn{2}{|l|}{ Year of ECMO treatment } \\
\hline $2005-2008$ & $1,400(6.6)$ \\
\hline 2009-2012 & $4,660(22.1)$ \\
\hline 2013-2015 & $6,640(31.4)$ \\
\hline $2016-2018$ & $8,429(39.9)$ \\
\hline \multicolumn{2}{|l|}{ Annual income level at ECMO treatment } \\
\hline Q1 (Lowest) or unknown & $5,222(24.7)$ \\
\hline Q2 & $3,809(18.0)$ \\
\hline Q3 & $4,849(22.9)$ \\
\hline Q4 (Highest) & $7,249(34.3)$ \\
\hline \multicolumn{2}{|l|}{ Case volume of ECMO treatment for 14 yrs } \\
\hline Q1<190 & $5,154(24.4)$ \\
\hline Q2: 190-393 & $5,518(26.1)$ \\
\hline Q3: 394-883 & $5,740(27.2)$ \\
\hline Q4 >883 & $4,717(22.3)$ \\
\hline Charlson comorbidity index, median [IQR] & $4[2,6]$ \\
\hline Length of hospital stay, day, median [IQR] & $13[5,28]$ \\
\hline
\end{tabular}

Table 1 (continued)
Table 1 (continued)

\begin{tabular}{|c|c|}
\hline Variable & Number (\%) \\
\hline Duration of ECMO therapy, day, median [IQR] & $4[1,9]$ \\
\hline Starting ECMO therapy on weekend & $4,647(22.0 \%)$ \\
\hline Monday & $3,870(18.3)$ \\
\hline Tuesday & $3,297(15.6)$ \\
\hline Wednesday & $3,243(15.3)$ \\
\hline Thursday & $3,138(14.9)$ \\
\hline Friday & $2,934(13.9)$ \\
\hline Saturday & $2,199(10.4)$ \\
\hline Sunday & $2,448(11.6)$ \\
\hline \multicolumn{2}{|l|}{ Main diagnosis at ECMO treatment } \\
\hline Cardiovascular disease & $11,130(52.7)$ \\
\hline ARDS or respiratory failure & $2,616(12.4)$ \\
\hline Post-cardiac arrest & $1,781(8.4)$ \\
\hline Cancer & $2,011(9.5)$ \\
\hline Gastrointestinal disease & $1,248(5.9)$ \\
\hline Sepsis & $566(2.7)$ \\
\hline Shock & $388(1.8)$ \\
\hline Trauma, burn, and injury & $422(2.0)$ \\
\hline Others & $967(4.6)$ \\
\hline
\end{tabular}

weekend group was 4,647 (22.0\%). Comparisons of clinicoepidemiological characteristics between the weekend and weekday groups are presented in Table 2. The weekend group was younger than the weekday group [median: 60 years (IQR, 49-70 years) and 61 years (IQR, $50-71$ years), respectively, $\mathrm{P}=0.018]$, and the proportion of male patients was higher on the weekend $(68.1 \%)$ than on weekdays $(65.7 \%)$ group $(\mathrm{P}=0.002)$. The $\mathrm{CCI}$ in the weekday group was higher than that in the weekend group [median: 4 (IQR, 2-6) and 3 (IQR, 2-6), respectively, $\mathrm{P}=0.036]$. No other clinico-epidemiological characteristics differed significantly between the groups.

\section{Sixty-day mortality risk and weekend admission}

Table S2 and Table 3 present the results of univariable and multivariable Cox regression analyses of 60-day mortality 
Table 2 Comparisons of clinico-epidemiological characteristics between the weekend and weekday groups

\begin{tabular}{|c|c|c|c|}
\hline Variable & Weekend group, $n=4,647$ & Weekday group, $n=16,482$ & $P$ value \\
\hline Sex, male & $3,165(68.1)$ & $10,821(65.7)$ & 0.002 \\
\hline Residence at ECMO treatment & & & 0.669 \\
\hline Capital city (Seoul) & $1,121(24.1)$ & $3,872(23.5)$ & \\
\hline Other area & $2,469(53.1)$ & $8,823(53.5)$ & \\
\hline Annual income level at ECMO treatment & & & 0.792 \\
\hline Q1 (Lowest) or unknown & $1,140(24.5)$ & $4,082(24.8)$ & \\
\hline Q2 & $861(18.5)$ & $2,948(17.9)$ & \\
\hline Year of ECMO treatment & & & 0.201 \\
\hline 2005-2008 & $292(6.3)$ & $1,108(6.7)$ & \\
\hline 2009-2012 & $1,054(22.7)$ & $3,606(21.9)$ & \\
\hline $2013-2015$ & $1,415(30.4)$ & $5,225(31.7)$ & \\
\hline 2016-2018 & $1,886(40.6)$ & $6,543(39.7)$ & \\
\hline Case volume of ECMO treatment for $14 \mathrm{yrs}$ & & & 0.092 \\
\hline Q1 $<190$ & $1,135(24.4)$ & $4,019(24.4)$ & \\
\hline Q2: 190-393 & $1,207(26.0)$ & $4,311(26.2)$ & \\
\hline 60-day mortality & $2,860(61.5)$ & $9,965(60.5)$ & 0.181 \\
\hline Main diagnosis at ECMO treatment & & & 0.112 \\
\hline Cardiovascular disease & $2,403(51.7)$ & $8,727(52.9)$ & \\
\hline ARDS or respiratory failure & $577(12.4)$ & $2,039(12.4)$ & \\
\hline Post-cardiac arrest & $431(9.3)$ & $1,350(8.2)$ & \\
\hline Cancer & $436(9.4)$ & $1,575(9.6)$ & \\
\hline Gastrointestinal disease & $261(5.6)$ & $987(6.0)$ & \\
\hline Sepsis & $145(3.1)$ & $421(2.6)$ & \\
\hline Shock & $95(2.0)$ & $293(1.8)$ & \\
\hline Trauma, burn, and injury & $90(1.9)$ & $332(2.0)$ & \\
\hline Others & $209(4.5)$ & $758(4.6)$ & \\
\hline
\end{tabular}

Presented as number with percentage or median value with interquartile range. ECMO, extracorporeal membrane oxygenation; ARDS, acute respiratory distress syndrome. 
Table 3 Multivariate Cox regression analyses of 60-day mortality risk among ECMO patients

\begin{tabular}{lcc}
\hline Variable & $\begin{array}{c}\text { Cox regression } \\
\text { model, HR }(95 \% \mathrm{Cl})\end{array}$ & P value \\
\hline
\end{tabular}

Weekend group (vs. weekday

group; model 1)

$1.05(1.01,1.09) \quad 0.025$

Sensitivity analysis (model 2)

\begin{tabular}{lcc} 
Wednesday & 1 \\
Thursday & $1.02(0.95,1.08)$ & 0.652 \\
Friday & $1.06(0.99,1.13)$ & 0.086 \\
Saturday & $1.11(1.04,1.19)$ & 0.003 \\
Sunday & $1.01(0.95,1.08)$ & 0.709 \\
Monday & $1.00(0.94,1.06)$ & 0.873 \\
Tuesday & $0.99(0.93,1.05)$ & 0.750 \\
Age, year & $1.01(1.01,1.02)$ & $<0.001$ \\
Sex, male & $0.97(0.99,1.01)$ & 0.111 \\
Residence at ECMO treatment & 1 & \\
Capital city (Seoul) & $1.07(1.02,1.11)$ & 0.004 \\
Other metropolitan city & $1.00(0.95,1.04)$ & 0.849 \\
Other area & $1.24(1.14,1.33)$ & $<0.001$ \\
Year of ECMO treatment & $1.01(0.93,1.09)$ & 0.882 \\
2005-2008 & $1.29(1.20,1.40)$ & $<0.001$ \\
2009-2012 & & \\
2013-2015 & & \\
2016-2018 & 1.24 & \\
\hline
\end{tabular}

Annual income level at ECMO treatment

$\begin{array}{lcc}\text { Q1 (lowest) or unknown } & 1 & \\ \text { Q2 } & 1.01(0.97,1.06) & 0.576 \\ \text { Q3 } & 1.01(0.96,1.07) & 0.671 \\ \text { Q4 (highest) } & 0.98(0.93,1.02) & 0.295\end{array}$

Case volume of ECMO treatment for $14 \mathrm{yrs}$

$\begin{array}{lcc}\text { Q1 }<190 & 1 & \\ \text { Q2: 190-393 } & 0.94(0.90,0.99) & 0.015 \\ \text { Q3: 394-883 } & 0.78(0.74,0.82) & <0.001 \\ \text { Q4 >883 } & 0.66(0.63,0.70) & <0.001 \\ \text { Charlson comorbidity index } & 1.10(1.10,1.11) & <0.001\end{array}$

Table 3 (continued)
Table 3 (continued)

\begin{tabular}{lcc}
\hline Variable & $\begin{array}{c}\text { Cox regression } \\
\text { model, HR }(95 \% \mathrm{Cl})\end{array}$ & P value \\
\hline $\begin{array}{l}\text { Duration of ECMO therapy, } \\
\text { day }\end{array}$ & $0.99(0.99,0.99)$ & $<0.001$ \\
Main diagnosis at ECMO treatment & 1 & \\
Cardiovascular disease & $0.95(0.89,1.00)$ & 0.058 \\
ARDS or respiratory failure & $1.99(1.88,2.11)$ & $<0.001$ \\
Post-cardiac arrest & $0.60(0.56,0.65)$ & $<0.001$ \\
Cancer & $0.46(0.41,0.51)$ & $<0.001$ \\
Gastrointestinal disease & $1.26(1.14,1.40)$ & $<0.001$ \\
Sepsis & $1.56(1.38,1.75)$ & $<0.001$ \\
Shock & $1.14(1.01,1.29)$ & 0.037 \\
Trauma, burn, and injury & $1.13(1.04,1.23)$ & 0.005 \\
\hline
\end{tabular}

ECMO, extracorporeal membrane oxygenation; HR, hazard ratio; $\mathrm{Cl}$, confidence interval; ARDS, acute respiratory distress syndrome; CPR, cardiopulmonary resuscitation.

risk among ECMO patients. In the multivariable Cox regression model, the weekend group had a higher risk of 60-day mortality than the weekday group (HR: 1.05, 95\% CI: 1.01-1.09; $\mathrm{P}=0.025)$. In sensitivity analysis, ECMO patients who had ECMO therapy initiated on a Saturday had an $11 \%$ higher risk of 60 -day mortality than those who had ECMO therapy initiated on a Wednesday (HR: 1.11, 95\% CI: 1.04-1.19; $\mathrm{P}=0.003)$; however, starting ECMO therapy on other days (Thursday, Friday, Sunday, Monday, or Tuesday) was not associated with an increased 60-day mortality risk (Figure 2). The C-index of the multivariable model was 0.87 (95\% CI: $0.86-0.88$ ), indicating that the model was highly predictive of 60-day mortality.

\section{Discussion}

This population-based cohort study showed that starting ECMO therapy on a weekend day was associated with a 60-day mortality risk that was slightly higher than that associated with starting ECMO therapy on a weekday. In the sensitivity analysis, this association was particularly evident in patients who received ECMO therapy starting on Saturday. This is the first nationwide cohort study of the weekend effect in the initiation of ECMO therapy in 

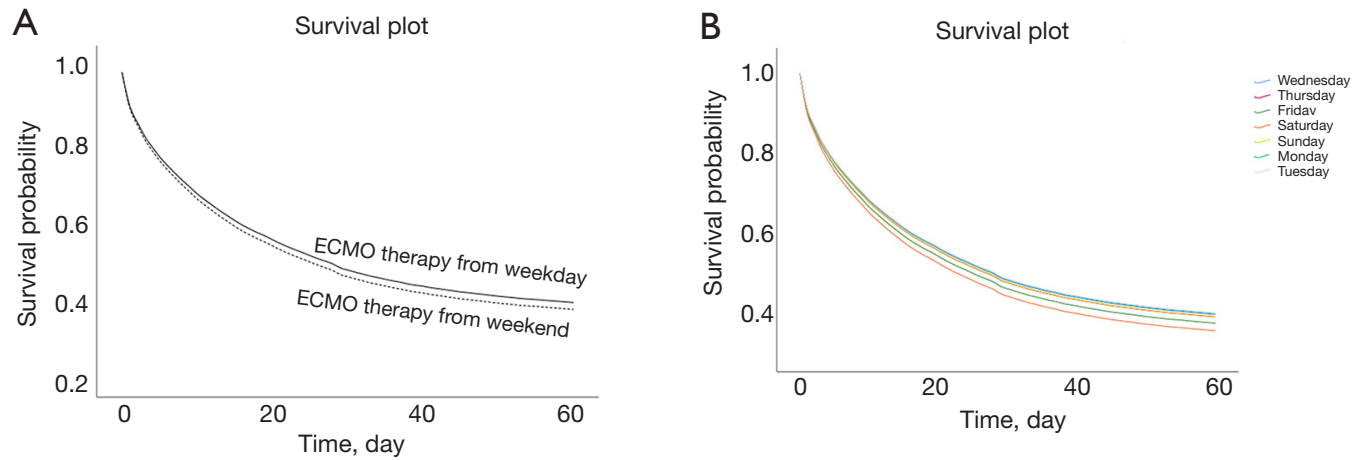

Figure 2 Sixty-day survival estimates, derived from multivariable Cox regression models: (A) Initiation of ECMO therapy during the weekend vs. weekday and (B) Initiation of ECMO therapy on a Thursday, Friday, Saturday, Sunday, Monday, and Tuesday vs. Wednesday. ECMO, extracorporeal membrane oxygenation.

South Korea. This study has shown that the differences in mortality risk between the weekend and weekday groups were independent of patient characteristics, which were similar between the groups (Table 2). As the number of clinical indications for ECMO therapy and its adoption are increasing (10), the present study findings may be relevant to improving survival outcomes in this patient group.

ECMO therapy is associated with complications that may result in subsequent adverse outcomes (18). A recent review article reported complications following ECMO therapy, including renal failure requiring continuous venovenous (VV) hemofiltration (52\%), bleeding (33\%), pneumonia $(33 \%)$, sepsis $(26 \%)$, oxygenator dysfunction requiring replacement (29\%), hemolysis (18\%), liver dysfunction $(16 \%)$, venous thrombosis $(10 \%)$, leg ischemia $(10 \%)$, central nervous system complications (8\%), gastrointestinal bleeding (7\%), disseminated intravascular coagulation (5\%), and aspiration pneumonia (5\%) (23). These complications occur primarily in patients who received veno-arterial (VA) ECMO therapy. However, it has been reported that $40.2 \%$ of patients who received VV-ECMO experience complications such as bleeding $(29.3 \%)$, intracranial hemorrhage $(5.4 \%)$, deep vein thrombosis/pulmonary embolism (6.4\%), pneumothorax (5.7\%), cannula infection (9.9\%), oxygenator failure $(12.8 \%)$, and cannula failure (4.5\%) (24).

These complications have been reported to have a close relationship with acute inflammatory reactions that occur in the early period after initiation of ECMO therapy (18). When ECMO therapy is initiated, the blood is exposed to extracorporeal circulation, which results in the activation of the humoral and cellular immunity response (25). This is a rapid process that involves coagulation factors such as
Factor XIIa, which reaches maximal levels in the ECMO circuit within 10 minutes of initiation (26). In addition to promoting coagulation factor activation, kallikrein production during ECMO therapy directly activates neutrophils, while bradykinin stimulates the release of nitric oxide, tumor necrosis factor-alpha, and interleukin 10 (26,27). Thus, the initiation of ECMO therapy might cause a systemic inflammatory response syndrome-like reaction, which might lead to endothelial cell activation (25). Some critical complications such as renal failure, sepsis, and pneumonia might be caused by this systemic reaction after the initiation of ECMO therapy (25); therefore, close monitoring and proper treatment are essential for patients undergoing ECMO therapy. In our study, the initiation of ECMO therapy on weekends, in particular, on Saturdays, was associated with mortality risk higher than that associated with ECMO therapy initiation on weekdays, suggesting that close monitoring or proper management during the weekend might be insufficient.

ICU staffing patterns in South Korea may partly account for these findings. In 2014, a survey of 220 ICUs at nine hospitals in South Korea, revealed that 38 (17.3\%) ICUs employed intensive care specialists on a 5 -weekday work week basis, and only 17 (7.7\%) employed fulltime adult critical care subspecialists (28), suggesting that most ICUs in South Korea might not have sufficient staff of experienced intensivists available for weekend shifts. In addition, a separate analysis reported that there was relatively poor ICU staffing in areas outside the capital city (Seoul) $(16,28)$. A recent study reported that a dedicated 24/7 ECMO service using a multidisciplinary team and defined protocols was able to reduce the rate of complications due to VA-ECMO therapy in patients with 
refractory cardiogenic shock (29). Overall, the weekendweekday discrepancies in ICU staffing in South Korea may affect the quality of ECMO management and monitoring, and the associated outcomes. However, the assessment of intensivist staffing patterns in South Korea and their impact on patient outcomes was outside the scope of this study, precluding any meaningful discussions on the impact of ICU staff on patient outcomes following ECMO therapy.

The present study has several limitations. First, patient characteristics such as body mass index, and smoking and drinking status were not considered, as the NHIS database does not record this type of information. Second, the disease severity of patients undergoing ECMO was neither evaluated nor confirmed through objective methods such as the Acute Physiology and Chronic Health Disease Classification System II or Simplified Acute Physiology Score II. Third, although we collected and adjusted for some confounders in this study, there may still be residual and unmeasured confounders that might affect the results. Lastly, we did not distinguish between VA-ECMO and VV-ECMO in this study due to the limitations of the ECMO prescription codes used in South Korea. As a result, the specific relevance of the present findings to patients undergoing each type of ECMO therapy cannot be ascertained.

\section{Conclusions}

This population-based cohort study in South Korea showed that weekend initiation of ECMO therapy was associated with a slightly increased 60-day mortality risk compared to that associated with weekday initiation of this therapy. This effect was particularly evident in patients who received ECMO therapy starting on a Saturday. This is the first study to report the evidence of the weekend effect in ECMO therapy initiation. Future studies are needed to confirm these findings.

\section{Acknowledgments}

Funding: None.

\section{Footnote}

Reporting Checklist: The authors have completed the STROBE reporting checklist. Available at http://dx.doi. org/10.21037/atm-21-180
Conflicts of Interest: All authors have completed the ICMJE uniform disclosure form (available at http://dx.doi. org/10.21037/atm-21-180). The authors have no conflict of interest to declare.

Ethical Statement: The authors are accountable for all aspects of the work in ensuring that questions related to the accuracy or integrity of any part of the work are appropriately investigated and resolved. The study was conducted in accordance with the Declaration of Helsinki (as revised in 2013). The study was approved by Institutional Review Board of Seoul National University Bundang Hospital (X-2001-586-902). The informed consent requirement was waived, as the data were anonymized and analyzed retrospectively, using a dataset extracted from the South Korean National Health Insurance Service (NHIS) database.

Open Access Statement: This is an Open Access article distributed in accordance with the Creative Commons Attribution-NonCommercial-NoDerivs 4.0 International License (CC BY-NC-ND 4.0), which permits the noncommercial replication and distribution of the article with the strict proviso that no changes or edits are made and the original work is properly cited (including links to both the formal publication through the relevant DOI and the license). See: https://creativecommons.org/licenses/by-nc-nd/4.0/.

\section{References}

1. Gattinoni L, Carlesso E, Langer T. Clinical review: Extracorporeal membrane oxygenation. Crit Care 2011;15:243.

2. Combes A, Hajage D, Capellier G, et al. Extracorporeal Membrane Oxygenation for Severe Acute Respiratory Distress Syndrome. N Engl J Med 2018;378:1965-75.

3. Lafc G, Budak AB, Yener AU, et al. Use of extracorporeal membrane oxygenation in adults. Heart Lung Circ 2014;23:10-23.

4. Tramm R, Ilic D, Davies AR, et al. Extracorporeal membrane oxygenation for critically ill adults. Cochrane Database Syst Rev 2015;1:CD010381.

5. Eckman PM, Katz JN, El Banayosy A, et al. Veno-Arterial Extracorporeal Membrane Oxygenation for Cardiogenic Shock: An Introduction for the Busy Clinician. Circulation 2019;140:2019-37.

6. Brodie D, Slutsky AS, Combes A. Extracorporeal Life 
Support for Adults With Respiratory Failure and Related Indications: A Review. JAMA 2019;322:557-68.

7. Aneman A, Brechot N, Brodie D, et al. Advances in critical care management of patients undergoing cardiac surgery. Intensive Care Med 2018;44:799-810.

8. Yang X, Cai S, Luo Y, et al. Extracorporeal Membrane Oxygenation for Coronavirus Disease 2019-Induced Acute Respiratory Distress Syndrome: A Multicenter Descriptive Study. Crit Care Med 2020;48:1289-95.

9. Barbaro RP, MacLaren G, Boonstra PS, et al. Extracorporeal membrane oxygenation support in COVID-19: an international cohort study of the Extracorporeal Life Support Organization registry. Lancet 2020;396:1071-8.

10. Mosier JM, Kelsey M, Raz Y, et al. Extracorporeal membrane oxygenation (ECMO) for critically ill adults in the emergency department: history, current applications, and future directions. Crit Care 2015;19:431.

11. Honeyford K, Cecil E, Lo M, et al. The weekend effect: does hospital mortality differ by day of the week? A systematic review and meta-analysis. BMC Health Serv Res 2018;18:870.

12. Cram P, Hillis SL, Barnett M, et al. Effects of weekend admission and hospital teaching status on in-hospital mortality. Am J Med 2004;117:151-7.

13. Meynaar IA, van der Spoel JI, Rommes JH, et al. Off hour admission to an intensivist-led ICU is not associated with increased mortality. Crit Care 2009;13:R84.

14. Arulkumaran N, Harrison DA, Brett SJ. Association between day and time of admission to critical care and acute hospital outcome for unplanned admissions to adult general critical care units: cohort study exploring the 'weekend effect'. Br J Anaesth 2017;118:112-22.

15. Galloway M, Hegarty A, McGill S, et al. The Effect of ICU Out-of-Hours Admission on Mortality: A Systematic Review and Meta-Analysis. Crit Care Med 2018;46:290-9.

16. Lim CM, Kwak SH, Suh GY, et al. Critical Care In Korea: Present and Future. J Korean Med Sci 2015;30:1540-4.

17. Davies MG, Hagen PO. Systemic inflammatory response syndrome. Br J Surg 1997;84:920-35.

18. Chen Q, Yu W, Shi J, et al. The effect of venovenous extra-corporeal membrane oxygenation (ECMO) therapy on immune inflammatory response of cerebral tissues in porcine model. J Cardiothorac Surg 2013;8:186.

19. Makdisi G, Wang IW. Extra Corporeal Membrane Oxygenation (ECMO) review of a lifesaving technology. J
Thorac Dis 2015;7:E166-76.

20. Lee DS, Chung CR, Jeon K, et al. Survival After Extracorporeal Cardiopulmonary Resuscitation on Weekends in Comparison With Weekdays. Ann Thorac Surg 2016;101:133-40.

21. von Elm E, Altman DG, Egger M, et al. The Strengthening the Reporting of Observational Studies in Epidemiology (STROBE) Statement: guidelines for reporting observational studies. Int J Surg 2014;12:1495-9.

22. Barbaro RP, Odetola FO, Kidwell KM, et al. Association of hospital-level volume of extracorporeal membrane oxygenation cases and mortality. Analysis of the extracorporeal life support organization registry. Am J Respir Crit Care Med 2015;191:894-901.

23. Zangrillo A, Landoni G, Biondi-Zoccai G, et al. A metaanalysis of complications and mortality of extracorporeal membrane oxygenation. Crit Care Resusc 2013;15:172-8.

24. Vaquer S, de Haro C, Peruga P, et al. Systematic review and meta-analysis of complications and mortality of veno-venous extracorporeal membrane oxygenation for refractory acute respiratory distress syndrome. Ann Intensive Care 2017;7:51.

25. Wachtfogel YT, Hack CE, Nuijens JH, et al. Selective kallikrein inhibitors alter human neutrophil elastase release during extracorporeal circulation. Am J Physiol 1995;268:H1352-7.

26. Larsson M, Rayzman V, Nolte MW, et al. A factor XIIa inhibitory antibody provides thromboprotection in extracorporeal circulation without increasing bleeding risk. Sci Transl Med 2014;6:222ra17.

27. Rodell TC, Naidoo Y, Bhoola KDJCI. Role of kinins in inflammatory responses. 1995;3:352-61.

28. Kwak SH, Jeong CW, Lee SH, et al. Current status of intensive care units registered as critical care subspecialty training hospitals in Korea. J Korean Med Sci 2014;29:431-7.

29. Koerner MM, Harper MD, Gordon CK, et al. Adult cardiac veno-arterial extracorporeal life support (VA-ECMO): prevention and management of acute complications. Ann Cardiothorac Surg 2019;8:66-75.

Cite this article as: Cho HW, Song IA, Oh TK. Weekend effect in extracorporeal membrane oxygenation therapy initiation: a nationwide cohort study in South Korea. Ann Transl Med 2021;9(9):742. doi: 10.21037/atm-21-180 


\section{Supplementary}

Table S1 The ICD-10 codes used by comorbidity to compute the Charlson comorbidity index

The ICD-10 codes used by comorbidity to compute the Charlson comorbidity index are:

Myocardial infarction: I21.x, I22.x, 125.2

Congestive heart failure: 109.9, I11.0, I13.0, I13.2, I25.5, 142.0, 142.5 - 142.9, 143.x, 150.x, P29.0

Peripheral vascular disease: I70.x, I71.x, I73.1, I73.8, I73.9, I77.1, I79.0, I79.2, K55.1, K55.8, K55.9, Z95.8, Z95.9

Cerebrovascular disease: G45.x, G46.x, H34.0, I60.x - I69.x

Dementia: F00.x - F03.x, F05.1, G30.x, G31.1

Chronic pulmonary disease: I27.8, I27.9, J40.x - J47.x, J60.x - J67.x, J68.4, J70.1, J70.3

Rheumatic disease: M05.x, M06.x, M31.5, M32.x - M34.x, M35.1, M35.3, M36.0

Peptic ulcer disease: K25.x - K28.x

Mild liver disease: B18.x, K70.0 - K70.3, K70.9, K71.3 - K71.5, K71.7, K73.x, K74.x, K76.0, K76.2 - K76.4, K76.8, K76.9, Z94.4

Diabetes without chronic complication: E10.0, E10.1, E10.6, E10.8, E10.9, E11.0, E11.1, E11.6, E11.8, E11.9, E12.0, E12.1, E12.6, E12.8, E12.9, E13.0, E13.1, E13.6, E13.8, E13.9, E14.0, E14.1, E14.6, E14.8, E14.9

Diabetes with chronic complication: E10.2 - E10.5, E10.7, E11.2 - E11.5, E11.7, E12.2 - E12.5, E12.7, E13.2 - E13.5, E13.7, E14.2 E14.5, E14.7

Hemiplegia or paraplegia: G04.1, G11.4, G80.1, G80.2, G81.x, G82.x, G83.0 - G83.4, G83.9

Renal disease: I12.0, I13.1, N03.2 - N03.7, N05.2 - N05.7, N18.x, N19.x, N25.0, Z49.0 - Z49.2, Z94.0, Z99.2

Any malignancy, including lymphoma and leukaemia, except malignant neoplasm of skin: C00.x - C26.x, C30.x - C34.x, C37.x - C41.x, C43.x, C45.x - C58.x, C60.x - C76.x, C81.x - C85.x, C88.x, C90.x - C97.x

Moderate or severe liver disease: 185.0, 185.9, I86.4, 198.2, K70.4, K71.1, K72.1, K72.9, K76.5, K76.6, K76.7

Metastatic solid tumour: C77.x - C80.x

AIDS/HIV: B20.x - B22.x, B24.x

Table S2 Univariable Cox regression analysis for 60-day mortality

\begin{tabular}{lcc}
\hline \multirow{2}{*}{ Variable } & Unadjusted Cox analysis & $\mathrm{HR}(95 \% \mathrm{Cl})$ \\
\cline { 2 - 2 } & $1.04(1.00,1.08)$ & 0.088 \\
Weekend group (vs weekday group) & & 1 \\
Sensitivity analysis & $1.02(0.96,1.09)$ & 0.459 \\
Wednesday & $1.05(0.98,1.12)$ & 0.155 \\
Thursday & $1.11(1.03,1.09)$ & 0.004 \\
Friday & $1.00(0.94,1.07)$ & 0.956 \\
Saturday & $1.00(0.94,1.06)$ & 0.960 \\
Sunday & $1.00(0.94,1.07)$ & 0.992 \\
Monday & & \\
\hline Tuesday & & \\
\hline
\end{tabular}

$\mathrm{HR}$, hazard ratio; $\mathrm{Cl}$, confidence interval 